\title{
Les places traditionnelles de Porto-Novo (Bénin) comme communs : entre mise en valeur des espaces urbains et promotion des pratiques sociales et culturelles
}

Traditional places as commons in Porto-Novo (Bénin): renovation of urban spaces and valorisation of social and cultural practices

\section{Elizabeth Auclair et Élise Garcia}

\section{OpenEdition Journals}

\section{Édition électronique}

URL : http://journals.openedition.org/developpementdurable/13147

DOI : 10.4000/developpementdurable. 13147

ISSN : 1772-9971

\section{Éditeur}

Association DD\&T

\section{Référence électronique}

Elizabeth Auclair et Élise Garcia, « Les places traditionnelles de Porto-Novo (Bénin) comme communs : entre mise en valeur des espaces urbains et promotion des pratiques sociales et culturelles », Développement durable et territoires [En ligne], Vol. 10, n¹ | Avril 2019, mis en ligne le 04 avril 2019, consulté le 01 mai 2019. URL : http://journals.openedition.org/ developpementdurable/13147 ; DOI : 10.4000/developpementdurable.13147

Ce document a été généré automatiquement le 1 mai 2019.

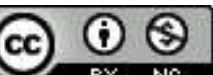

Développement Durable et Territoires est mis à disposition selon les termes de la licence Creative Commons Attribution - Pas d'Utilisation Commerciale 4.0 International. 


\title{
Les places traditionnelles de Porto- Novo (Bénin) comme communs : entre mise en valeur des espaces urbains et promotion des pratiques sociales et culturelles
}

\author{
Traditional places as commons in Porto-Novo (Bénin): renovation of urban \\ spaces and valorisation of social and cultural practices
}

Elizabeth Auclair et Élise Garcia

1 Une grande fête a eu lieu le samedi 9 janvier 2016 dans la ville de Porto-Novo, au Bénin, afin d'inaugurer les deux places traditionnelles Azalou comè et Djihoué comè, qui ont fait l'objet d'une opération de réhabilitation, articulant mise en valeur du patrimoine et création artistique. L'opération a été initiée par la municipalité de Porto-Novo et la Communauté d'agglomération de Cergy-Pontoise (CACP), dans le cadre de la coopération décentralisée mise en œuvre depuis 1995 entre ces deux collectivités territoriales.

Porto-Novo, la capitale du Bénin, est située au sud du pays, au bord d'une lagune ouvrant sur l'océan Atlantique. La ville, qui compte environ 300000 habitants $^{1}$, est restée jusqu'à présent à l'écart des phénomènes d'urbanisation galopante que connaissent les autres capitales africaines. Néanmoins, le développement du corridor économique de la côte ouest africaine entre Lagos (Nigeria) et Abidjan (Côte d'Ivoire) influe sur l'évolution de Porto-Novo. Le centre historique et colonial est actuellement en déclin et perd des habitants au profit des quartiers périphériques marqués par l'étalement urbain (Dorier et al., 2013). La ville est localisée à une trentaine de kilomètres de Cotonou ${ }^{2}$, qui grâce à son port et son ouverture maritime est devenue depuis les années cinquante la capitale économique du pays, et à ce titre rassemble un grand nombre de structures économiques, administratives et politiques (N’Bessa, 2013). 
3 L'opération de réhabilitation des places, appelée "Éclosions urbaines», se présente comme « à la croisée de l'urbanisme, de l'art public, du design urbain et de l'économie urbaine de proximité $e^{3} »$. L'approche choisie pour réhabiliter ces places repose sur la participation des habitants, des usagers, des artisans et des artistes de la ville. Le projet a ainsi été conçu comme une "opération pilote ", en quelque sorte, permettant ensuite de poursuivre, en fonction des opportunités de financement, l'aménagement des autres places traditionnelles de la ville. Porto-Novo comprend en effet plus d'une cinquantaine de places traditionnelles dont beaucoup sont ignorées ou menacées (Bassalé, 2014). Une étude récente a permis d'identifier 19 places susceptibles de bénéficier d'une telle réhabilitation ${ }^{4}$. D'ores et déjà, l'Agence française de développement (AFD) envisage de financer la réhabilitation de cinq places, dans le cadre du projet d'aménagement « PortoNovo - Ville verte $»^{5}$.

4 Ces places dites traditionnelles jouent un rôle fondamental pour les collectivités familiales locales, c'est-à-dire les familles élargies vivant autour de ces places, mais aussi les membres de la famille vivant dans d'autres villes et villages. Elles détiennent un statut particulier dans l'espace urbain, combinant des dimensions à la fois privées, publiques et « communes ». En effet, ces places «appartiennent» symboliquement ${ }^{6}$ au lignage, à la famille au sens élargi, qui vit autour de la place, mais aussi plus largement aux personnes liées à cette place pour des raisons historiques, familiales ou spirituelles. Les formes d'appartenance et d'attachement aux places sont complexes: il existe à la fois un attachement que l'on peut qualifier de familial, générationnel, aux espaces où les ancêtres se sont installés et ont vécu (on parle du lignage), et un attachement que l'on peut qualifier de cultuel et culturel à ces lieux, où existent des arbres sacrés, des temples et autels dédiés aux divinités locales et familiales, et où se déroulent les pratiques et cérémonies vodoun, ainsi que les fêtes traditionnelles de la collectivité familiale comme les mariages et les baptêmes. La collectivité familiale englobe la famille élargie vivant autour de la place, mais aussi les membres de la famille vivant dans d'autres villes ou à l'étranger. Lieux importants en termes de pratiques religieuses, ces espaces sont aussi des lieux de sociabilité, de convivialité, de proximité familiale et de voisinage, à partir desquels divers usages culturels, sociaux et commerciaux se développent.

L'opération de réhabilitation des places traditionnelles de Porto-Novo semble un cadre pertinent pour questionner l'articulation entre les dimensions matérielles et immatérielles des communs. Nous avons analysé cette opération dans une recherche menée au sein du Laboratoire de géographie MRTE de l'université de Cergy-Pontoise, articulant aménagement et géographie sociale ${ }^{7}$. Après avoir présenté le cadre théorique et conceptuel de notre recherche ainsi que la problématique, nous exposerons les principales caractéristiques des places traditionnelles de Porto-Novo, pour ensuite analyser les perceptions et représentations de ces places issues des entretiens avec les habitants, et examiner les dynamiques participatives mises en œuvre lors de la réhabilitation. 


\section{Les communs à la croisée des enjeux d'aménagement urbain, de patrimonialisation et de participation des habitants}

6 À partir de la singularité du cas de Porto-Novo, cet article a pour ambition de contribuer aux recherches portant sur la notion complexe et polysémique des communs. L'objectif de cette contribution est d'analyser en quoi ces places constituent des communs, en étudiant la complexité de la vie sociale qui s'organise - et se réorganise - via l'aménagement et la mise en valeur des patrimoines, et de mettre en lumière l'articulation entre les dimensions matérielles et immatérielles qui caractérisent ces espaces urbains. Au-delà de son inscription dans le cadre théorique relatif aux communs, notre propos croise deux registres conceptuels concernant, d'une part, le patrimoine urbain et les processus de patrimonialisation, et, d'autre part, les démarches de participation des habitants et de co-construction de projets.

7 Le concept des communs revient en force depuis quelques années dans les débats théoriques et scientifiques (Bollier, 2014 ; Dardot et Laval, 2014 ; Coriat, 2015, Cornu et al., 2017, Leyronas et Bambridge, 2018), comme dans les projets qui se multiplient, portés par des collectivités territoriales ou par la société civile, fédérant des luttes contre les processus de privatisation et de marchandisation liés au modèle néolibéral. Ces mouvements se mobilisent pour la mise en œuvre de sociétés soutenables, répondant aux enjeux de préservation de l'environnement, d'équité sociale et de renforcement de la démocratie locale. Rappelons que les communs représentent une forme d'organisation sociale et collective pour la gestion de ressources fondamentales, qui existe depuis longtemps et perdure dans de nombreux pays du Sud, comme l'a montré Elinor Ostrom dans ses travaux (1990). Si de nombreuses analyses portent sur les ressources dites naturelles (l'eau, la forêt, les semences, la biodiversité, etc.), de multiples travaux concernent aujourd'hui les ressources immatérielles de la connaissance, du savoir, de la culture, notamment au travers du numérique (Le Crosnier, 2015). Par ailleurs des chercheurs s'intéressent à la dimension territoriale des communs, via le paysage (Sgard, 2010), la ville (Boniburini et al., 2013), ou le territoire lui-même (Magnaghi, 2014).

8 Nous proposons ici de compléter ces approches des communs en travaillant sur la ville, et plus précisément sur le patrimoine urbain. En effet, face à l'urbanisation croissante de la population de la planète, la question de la ville devient centrale dans les réflexions sur la soutenabilité des territoires. Dans le contexte actuel de mondialisation des flux, d'accroissement des mobilités, d'émergence de sociétés urbaines multiculturelles et de métropolisation des territoires, la question des identités et des appartenances est réinterrogée. Ainsi, les processus de patrimonialisation sont devenus un enjeu d'aménagement urbain, de par le monde, mais aussi un enjeu de mobilisations et de revendications sociales et culturelles (Auclair et al., 2017). David Harvey (2015) s'est intéressé à la question des communs urbains, en lien avec la problématique du droit à la ville. Il considère que la notion de droit à la ville (Lefebvre, 1967) est utile, dans la mesure où elle permet de poser un regard en termes politiques et économiques sur les évolutions urbaines actuelles. Harvey réinterroge ainsi cette notion de droit à la ville : de quelle ville s'agit-il ? Un droit, mais pour qui, pour quelles catégories sociales? Comment éviter la privatisation de la ville? Comment éviter l'appropriation privative du sol ? Si la ville est un bien commun par excellence, qui gère ce commun, et au bénéfice de qui ? Ces 
questionnements renvoient directement aux enjeux soulevés par les communs, et peuvent être mobilisés pour l'étude des places de Porto-Novo.

Selon David Bollier (2014), trois éléments qui se combinent permettent de définir un commun: l'existence d'une ressource fondamentale, l'identification d'un groupe pour lequel cette ressource est considérée comme essentielle, et la mise en œuvre d'un certain nombre de règles sociales collectives, autour d'un ensemble de valeurs, de pratiques et d'usages. Comme on peut le voir, la définition même des communs semble témoigner d'une articulation singulière entre la dimension matérielle et la dimension immatérielle de «l'objet » concerné.

Notre travail sur les places traditionnelles s'inscrit aussi dans les réflexions et travaux sur le patrimoine, marqués par l'extension chronologique, catégorielle et spatiale de cette notion (Heinich, 2009), et par la diffusion du paradigme participatif dans l'action publique. On assiste à un renouvellement des modalités de patrimonialisation et à une redéfinition de la notion de patrimoine, qui valorisent les " communautés patrimoniales » autant que les objets (Hertzog et al., 2017). Ces évolutions sont sous-tendues par un ensemble de textes internationaux qui recommandent un recentrage sur les personnes et les populations concernées par ces "objets patrimoniaux », et une reconnaissance de la diversité des cultures et des patrimoines. La convention Unesco de 2003 sur le patrimoine immatériel tout comme la convention Unesco de 2005 sur la protection et la promotion de la diversité des expressions culturelles conduisent à prendre davantage en compte la diversité des cultures, des modes de vie, des traditions et savoir-faire. Ces textes incitent les habitants à identifier, définir et mettre en valeur eux-mêmes les ressources de leur territoire - richesses environnementales, économiques, sociales, culturelles. La recommandation de l'Unesco de 2011 sur les paysages urbains historiques ainsi que la convention de Faro, de 2005, sur la valeur du patrimoine culturel pour la société, portée par le Conseil de l'Europe, vont encore plus loin dans ce recentrage sur les populations, en incitant au renforcement des processus participatifs, et dans l'élargissement de la notion, par la reconnaissance du patrimoine ordinaire, du patrimoine du quotidien (Auclair et Fairclough, 2015). Ce passage de la notion d'objet patrimonial à la notion de processus témoigne du renforcement des liens entre les dimensions matérielle et immatérielle des richesses locales, phénomène que l'on observe sur les places de Porto-Novo.

Ces nouvelles approches mettent aussi l'accent sur la dimension sensible, affective du patrimoine. De nombreux travaux de géographes, sociologues ou anthropologues ont été consacrés à l'étude de "ce qui compte", de "ce qui fait sens" pour les habitants (Veschambre, 2008; Gravari Barbas, 2003 ; Rautenberg, 2003 ; Watremez, 2008). Dans son travail sur les "émotions patrimoniales", Daniel Fabre (2013) souligne «la force des attachements locaux et des investissements émotionnels qu'il suscite ", ainsi que l'importance des histoires personnelles. L'ordinaire, le familier, l'intime sont pour lui des valeurs essentielles pour comprendre et analyser les processus contemporains de patrimonialisation (Fabre, 2016). Nous nous appuyons aussi sur les analyses de Laurajane Smith (2006), qui considère que le patrimoine est un processus complexe qui comprend des actes de commémoration et de mémoire, mais aussi la construction de sentiments d'attachements et d'appartenances, et qu'il participe de la construction du lieu, de l'espace, du territoire, et favorise la compréhension du présent. Selon elle, si le patrimoine peut signifier des choses différentes selon les personnes, il constitue néanmoins un processus qui construit des valeurs et des significations culturelles communes; d'où l'importance des démarches participatives permettant de s'extraire des 
" discours autorisés ", c'est-à-dire les discours portés exclusivement par les " experts ». Elle défend une approche du patrimoine en tant que pratique culturelle, ce qui suppose la construction et l'organisation d'un ensemble de valeurs et de représentations, dont l'authenticité repose sur le sens que les habitants lui donnent, dans leur vie quotidienne.

Dans la recherche sur les places traditionnelles de Porto-Novo, le rôle accordé au sensible et au symbolique dans les processus de patrimonialisation semble constituer le cadre d'analyse nous permettant d'explorer la notion des communs dans ses dimensions matérielle et immatérielle. Nous considérons que ces formes collectives d'appartenance et d'attachement au passé, et notamment aux traditions et pratiques cultuelles et culturelles, peuvent être comprises comme les signes de la construction de communs. Nous estimons que ce patrimoine peut ainsi être qualifié de ressource fondamentale pour les habitants, et c'est l'attachement de la population à cette "ressource", ainsi que l'organisation et la dynamique sociale qui l'entourent, qui permettent, selon nous, de le définir comme commun.

\section{Les places traditionnelles de Porto-Novo, au cœur d'une structure urbaine et sociale complexe}

13 Porto-Novo est le nom donné à la ville au xviII ${ }^{e}$ siècle par les Portugais, lesquels y installèrent un port et participèrent activement à la traite négrière. Entre le $\mathrm{XV}^{\mathrm{e}}$ siècle et la fin du XIX $x^{e}$ siècle, différents courants migratoires ont contribué au peuplement de ce territoire et à sa diversité culturelle, avec notamment deux principales communautés : les Yoruba issus du Nigeria et les Goun venant du Togo. Toutes ces populations ont eu pour caractéristique commune de pratiquer le culte vodoun (Sinou et Oloudé, 1989), ce qui contribue à définir la partie sud du Bénin comme le berceau de la culture vodoun (Tall, 1995 ; Cousin, 2013, Bassalé, 2014). Pendant les siècles du commerce triangulaire (du XVII ${ }^{\mathrm{e}}$ au XIX ${ }^{e}$ siècle), de nombreux esclaves ont été emmenés au Brésil, à Cuba et en Haïti, ce qui explique la diffusion des pratiques vodoun dans ces pays (Vidégla, 2013). Des similitudes entre les pratiques vodoun au Brésil et en Afrique ont été clairement mises en évidence (Verger, 1954; Tall, 2009). À la fin du XIX ${ }^{e}$ siècle, le pays devint une colonie française (l'ancien Dahomey) avant d'accéder à l'indépendance en 1960, gardant ainsi des traces dans l'architecture coloniale dans les villes.

14 La ville historique de Porto-Novo est reconnue pour la richesse de ses patrimoines matériel et immatériel (Sinou, 2013). Si l'accent a d'abord été mis sur le patrimoine architectural et bâti (notamment les bâtiments coloniaux et les édifices afro-brésiliens ${ }^{8}$ ), on remarque que d'autres types de patrimoine ont été mis en évidence plus récemment, comme le patrimoine naturel de la lagune ${ }^{9}$ ainsi que les arbres et forêts sacrés (Juhé Beaulaton, 2009). Actuellement, on voit émerger un intérêt grandissant, de la part des acteurs locaux, nationaux et internationaux, pour le patrimoine immatériel que constituent les pratiques et traditions vodoun ${ }^{10}$. Le réseau des places traditionnelles vodoun constitue la matrice de l'organisation spatiale et symbolique du tissu ancien de la ville, au sein duquel s'insèrent des bâtiments coloniaux et des bâtiments afro-brésiliens, dont beaucoup sont à l'abandon, voire en ruine. Les places traditionnelles font partie du patrimoine de la ville, même si elles sont souvent restées invisibles et méconnues. Les travaux de l'École du patrimoine africain (EPA) évoquent un patrimoine "vernaculaire ", un patrimoine « paysager » (Bassalé, 2014). Si l'on reprend les termes de l'Unesco ou du 
Conseil de l'Europe, on peut aussi parler de patrimoine ordinaire, familier, du quotidien. Pour comprendre le fonctionnement de ces places, nous pouvons nous appuyer sur les propos de l'urbaniste Bachir Oloudé (2003), qui propose une analyse du patrimoine existant dans les espaces urbains en Afrique : "Un arbre, un lieu, un chemin, un cours d'eau peuvent y remplir une fonction à la fois matérielle et immatérielle. L'invisible, accessible aux initiés, est le fondement réel de la cité. Ainsi même si l'événement matériel (lieu ou site culturel) n'a pas de valeur patrimoniale avérée, l'immatériel qu'il véhicule a une valeur essentielle dans la vie sociale. C'est cette interaction entre patrimoine matériel, visible, bâti, et patrimoine immatériel ou invisible qui forge la spécificité de la ville africaine. "

\subsection{Les rites et cérémonies vodoun, un patrimoine qui articule dimension matérielle et immatérielle}

$15 \mathrm{Au}$ cours de l'histoire, différentes communautés se sont installées autour de places, souvent choisies par l'existence de grands arbres (témoins de la présence d'eau, et symboles religieux). Ces groupes ont construit des temples et couvents vodoun, et de petits édifices et autels dédiés à leurs divinités. Les places vodoun de Porto-Novo ont régulièrement été menacées, voire détruites au cours de l'histoire. Pendant la période de la colonisation, des églises et des cathédrales, mais aussi des écoles ont parfois été construites sur ces places, et des routes ont été percées, répondant à un objectif de modernisation de la ville, tout en constituant pour les autorités une manière de lutter contre les pratiques religieuses indigènes, condamnées par l'Église catholique ${ }^{11}$. Ultérieurement, ces places ont également été menacées pendant le régime communiste de Mathieu Kérékou (de 1972 à 1989), lequel a interdit toutes les religions, y compris les pratiques vodoun. Mais si de nombreux prêtres vodoun ont été arrêtés et torturés, la plupart des habitants ont continué leurs pratiques, de manière discrète, chez eux (Bassalé, 2013). Depuis plus d'une vingtaine d'années, la pratique du culte vodoun a été autorisée et a désormais repris avec force, notamment dans le sud du pays et en particulier à Porto-Novo; une fête officielle et chômée, dédiée au vodoun, a même été institutionnalisée le 10 janvier, au titre de patrimoine culturel national.

Les liens entre les habitants du quartier s'établissent pour une grande part au travers des rites et cérémonies vodoun qui structurent les communautés. Ces pratiques - ou du moins l'attachement à ces traditions - sont encore fortement ancrées au sein de la population, y compris chez les jeunes générations, les artistes et les intellectuels, même si les chiffres précis des pratiquants sont difficiles à obtenir ${ }^{12}$. En effet, le culte vodoun n'est pas exclusif: il s'articule avec la pratique d'autres religions (Dorrier-Apprill, 2006). Être catholique, protestant ou musulman renvoie à une "religion », tandis que le culte vodoun semble davantage relever d'une pratique familiale, traditionnelle, ancestrale. Ainsi le culte vodoun paraît représenter tout à la fois une forme de pratique culturelle, une tradition, une mémoire collective, un patrimoine qu'il faut respecter, conserver et protéger. Comme le résume un habitant : "C'est notre patrimoine, c'est notre identité. Même les jeunes qui partent ailleurs, faire leurs études, restent attachés à cela. » 


\subsection{Les places traditionnelles, support de la vie cultuelle et culturelle locale}

17 L'espace du quotidien est désormais considéré comme un patrimoine à part entière. Christine Mengin (2013) définit le patrimoine urbain comme « résultant d'une stratification dans le temps de différents éléments formels : les édifices, monumentaux et ordinaires, les rues, les places, le tissu urbain. Il englobe aussi les aspects sociaux, économiques et liés à l'usage ». Elle considère que l'une des spécificités des villes africaines repose sur l'articulation entre le visible et l'invisible. On remarque que les places traditionnelles de Porto-Novo illustrent plusieurs de ces combinaisons: visible/invisible, public/privé, matériel/immatériel, sacré/profane. Les travaux de recherche menés par Victor Brunfaut et Sara Tassi, de l'Université libre de Bruxelles, sur les espaces publics des villes africaines, et notamment au Bénin, mettent en lumière les tensions entre les approches "traditionnelles" africaines et les approches laïques proposées par la modernité occidentale, ainsi que l'articulation entre les dimensions matérielle et immatérielle des statuts et des usages.

Les places traditionnelles de Porto-Novo sont généralement structurées autour d'un temple vodoun ouvert sur la place par un vestibule, à l'arrière duquel on accède à un couvent. Le temple est dédié à la principale divinité locale, qui «appartient» à une famille, une collectivité en particulier. Seuls les « adeptes $^{13}$ » et les « initiés » ont le droit d'y pénétrer. Sur les places traditionnelles, il existe aussi un ou plusieurs arbres sacrés ${ }^{14}$, protégés pour leur valeur religieuse, mais aussi pour leurs vertus médicinales (JuhéBeaulaton, 2009), ainsi qu'un certain nombre de petits édifices, autels et niches votives, les legba ${ }^{15}$, situés sur la place ou incrustés dans les façades des habitations ou des murs qui entourent la place. Différentes divinités liées aux éléments naturels (eau, terre, feu...) peuvent être honorées sur ces places, chacune ayant une signification particulière (Bassalé, 2014). Par ailleurs, il existe souvent un couvent pour abriter les zangbetos, considérés comme les " gardiens de la nuit ${ }^{16}$ ». Il existe une grande cérémonie, le hounwé, qui rassemble généralement une fois par an, tous les habitants de la place ainsi que les personnes de la famille, de la communauté. Les personnes vivant ailleurs, dans d'autres villages, voire à l'étranger, reviennent ici sur la place pour la célébration.

Ces places sont légalement considérées par l'administration territoriale comme des domaines publics (Bassalé, 2013). Néanmoins, elles sont organisées autour des pratiques vodoun de la collectivité familiale qui vit aux abords. Elles peuvent ainsi être considérées comme des places "communes", puisqu'elles appartiennent symboliquement à un groupe, une famille, un lignage. Mais elles ne sont pas privées, puisqu'elles sont ouvertes à tous, et qu'elles constituent des lieux de socialité et de convivialité pour le quartier : on peut les traverser, s'y promener, s'y arrêter, jouer aux cartes, se rendre chez le coiffeur, ou y manger dans les cuisines de rue et faire ses courses auprès des commerçantes (appelées «les bonnes dames»). La séparation entre les dimensions cultuelles et culturelles des traditions locales semble ténue : aux cérémonies et rites vodoun s'ajoutent en effet d'autres fêtes familiales traditionnelles (baptêmes, mariages et fêtes funéraires) organisées tout au long de l'année, avec des danses, musiques et chants. 


\subsection{Le projet de réhabilitation des places comme révélateur des communs}

20 territoire d'inventer le leur en fonction de leurs ressources disponibles qu'il s'agit de valoriser à partir de l'histoire et de la culture propre de chacun.» Cette analyse de Michel Vernières (2013, p. 19) peut s'appliquer à la démarche qui a été initiée pour la réhabilitation des places de Porto-Novo. L'existence de ressources à protéger et mettre en valeur constituant un élément central du concept des communs, on peut considérer que le patrimoine matériel et immatériel de ces places compose cette ressource jugée fondamentale par les habitants. Mais c'est moins sa dimension matérielle que sa dimension immatérielle qui semble importante pour les habitants, et comme le rappelle Saskia Cousin (2013) : «La revendication sociale ou identitaire n'est pas liée au patrimoine historique bâti.» On peut rappeler que l'usage du terme patrimoine - une notion occidentale - reste complexe, dans ce contexte. Comme le soulignent Didier Houénoudé et Monica Coralli (2013), on remarque souvent une forme d'indifférence de la part des habitants vis-à-vis des bâtiments anciens, qui se détériorent, voire disparaissent. En revanche, un intérêt majeur semble porté à la préservation des pratiques culturelles traditionnelles. C'est la raison pour laquelle l'opération de réhabilitation a pu être perçue comme exemplaire - et innovante -, puisqu'il s'agissait de travailler avec les habitants, pour préserver et mettre en valeur leurs pratiques culturelles, via les travaux sur les places. Cela explique en partie sans doute le sentiment général de satisfaction noté lors des entretiens.

21 Un des enjeux du projet d'aménagement a donc été de mobiliser les habitants afin de les rendre acteurs des transformations urbaines. Pour le directeur des relations internationales et du tourisme de la CACP, ayant activement soutenu le projet au titre de la coopération décentralisée, «l'urbaniste doit repositionner la question de l'expérience urbaine des habitants au coeur du processus de mutation qu'il veut accompagner» (Raimbault, 2015, p. 7). L'opération cherche ainsi à se distinguer des grands projets urbains et touristiques contemporains qui fleurissent dans de nombreuses villes du monde, et qui commencent à être envisagés aujourd'hui par le gouvernement béninois.

Deux places traditionnelles, la place Lokossa et la place Agonsa honto, avaient précédemment fait l'objet de réhabilitations. Le projet de la place Lokossa a été porté principalement par l'EPA et l'École d'architecture de la Cambre, en Belgique. Un deuxième projet a ensuite été initié en 2012 par l'Institut français et la Cité de l'architecture et du patrimoine à Paris, dans le cadre d'un projet plus large intitulé «Liaisons urbaines », dont l'objet était d'améliorer les espaces urbains et le cadre de vie des habitants dans trois villes situées à Porto-Novo, à N'Djaména et à Casablanca ${ }^{17}$. À Porto-Novo, l'opération visait plus particulièrement le développement des activités de restauration de rue; le projet a été porté par l'EPA et coordonné par Gérard Bassalé, directeur du Centre culturel Ouadada.

Suite à ces expériences, le projet intitulé « Éclosions urbaines » a été initié en 2013 par les villes de Porto-Novo et la CACP, et deux places ont été aménagées dans le cadre de la célébration des 20 ans de la coopération décentralisée. La ville de Porto-Novo a réalisé certains aménagements comme la réfection du réseau d'évacuation des eaux usées, le pavage d'une des places, et l'installation de luminaires, mais n'est pas porteuse du projet. C'est le directeur du centre culturel Ouadada qui au final a été le principal «maître

Développement durable et territoires, Vol. 10, n | Avril 2019 
d'œuvre» du projet ${ }^{18}$. La réhabilitation a commencé par les deux places «jumelles » Azalou comè et Djihoué comè (séparées par un escalier qui descend vers la lagune, lequel a également fait l'objet d'un réaménagement). L'opération a pour but de faire participer les habitants aux décisions et aux activités de rénovation, et de mobiliser les artistes plasticiens de la ville.

La participation des habitants apparaît comme une dimension fondamentale du projet: l'objectif était de s'appuyer sur les sentiments d'appartenance et d'attachement des habitants pour les impliquer dans les travaux, selon des modalités diverses. Le principe était aussi de permettre à la population locale d'origine modeste d'être rétribuée pour son investissement dans l'opération. Cette approche peut expliquer en partie la satisfaction exprimée par les habitants. Le quartier de Porto-Novo, dans lequel sont situées les places Azalou comè et Djihoué comè, localisé à l'ouest du centre-ville historique, proche de la lagune, abrite une population en situation de précarité plus ou moins marquée (Dorier et al., 2013). La situation économique de la ville s'étant dégradée ces dernières années (en raison de la récession au Nigeria voisin, et notamment de la faiblesse des cours du pétrole), beaucoup d'habitants, ne trouvant plus de travail correspondant à leurs qualifications, ont été amenés à occuper des emplois informels, parfois dangereux et souvent durs physiquement. Si le centre historique connaît une diminution de sa population, de nombreux habitants des quartiers concernés par l'étude sont nés dans le quartier et n'en ont jamais bougé. La précarisation de la population et le manque de moyens des habitants ont eu comme conséquence une dégradation marquée des espaces publics et du patrimoine des places.

L'approche participative mise en œuvre a permis de croiser différentes expertises : celles des urbanistes, des architectes, des maçons, des artistes, et celles des habitants. Ces derniers ont été mobilisés selon leurs compétences et leurs capacités, en renforçant d'une certaine manière leurs "capabilités " (Nussbaum, 2011). On peut considérer que cette démarche, qui redonne des fonctions et des rôles aux habitants, participe d'une forme d'extension du "droit à la ville », tel que le définissait Henri Lefebvre, élargi au droit au patrimoine, « un droit pour chacun de disposer d'un passé objectivé et localisé, hérité ou choisi ", selon les termes de Daniel Fabre (2013).

Ce processus transversal et inclusif semble constituer un exemple de coconstruction patrimoniale, dont l'objet est de remettre les habitants au cœur des décisions (Auclair et al., 2017). Pour Christine Mengin (2013, p. 30), « ces pratiques jouent un rôle essentiel dans la préservation du patrimoine culturel immobilier africain [...]. Elles témoignent que les activités de conservation, loin d'être l'apanage de spécialistes autorisés, sont le fait des communautés locales, qui considèrent la conservation du patrimoine comme une combinaison d'activités techniques et de protection des valeurs spirituelles $"$.

\section{L'appartenance et l'attachement, les éléments qui définissent ces places comme communs}

"J'y tiens comme à la prunelle de mes yeux »: la manière dont les habitants parlent de leurs places permet de prendre la mesure du sentiment d'appartenance et d'attachement qui les y lie. La majorité des personnes interrogées ont en effet souligné la très grande importance que revêtent ces lieux dans leur vie. Une dimension presque physique, voire biologique caractérise cet attachement, se traduisant par une personnification de la 
place, voire son intériorisation comme une composante à part entière de l'identité des habitants. Plusieurs facteurs contribuent à envisager ce patrimoine comme un commun tout à la fois matériel et immatériel : la dimension historique et familiale, la place centrale du culte vodoun et les autres usages collectifs de la place qui participent du lien social.

\subsection{La dimension historique et familiale des places}

Les habitants interrogés se désignent pour une large majorité comme étant » natifs d'ici ", et à ce titre héritiers d'une famille et d'un lignage, d'une histoire qu'il convient de perpétuer. Si cette place est si importante à leurs yeux, c'est également parce qu'y sont nées les générations précédentes. La notion d'héritage, comme un bien commun reçu à la naissance et dont le groupe est dépositaire, est très prégnante et prend la forme d'une responsabilité coportée par les générations actuelles et à venir. Cette transmission est avant tout orale, elle s'apprend des anciens ; la transmission est alimentée par un certain nombre de coutumes communes au groupe.

La notion d'appartenance peut revêtir un double sens: les habitants, qui se sentent appartenir à la place, ont également le sentiment que celle-ci leur appartient. À la question "à qui appartiennent ces places? ", certaines réponses des habitants sont en effet révélatrices : "Cette place est la nôtre, c'est notre patrimoine, c'est-à-dire qu'elle appartient $\grave{a}$ nous tous. Mes arrières-grands-parents, mes parents ont toujours dit que la terre n'appartient à personne, elle ne se vend pas, nous sommes nés et nous avons vu cette terre, ce sont les hommes qui occupent cette terre, c'est notre chose. » Les personnes interrogées s'accordent à dire que les places ne sont pas la propriété privée de quelqu'un: «C'est une propriété commune à tous les fils et filles de ce quartier ", indique l'une d'entre elles. Plusieurs personnes, percevant les places avant tout dans leur nature sacrée, considèrent qu'elles appartiennent aux divinités qui y sont installées.

\subsection{La dimension cultuelle de ces places traditionnelles}

Cet héritage commun, qui repose à la fois sur un espace et ses différentes composantes physiques (les habitations, les temples, les couvents, les arbres sacrés, etc.) et sur les pratiques culturelles et cultuelles, témoigne du lien entre les dimensions matérielles et immatérielles de ce patrimoine.

Tableau 1. Les dimensions matérielles et immatérielles du patrimoine

\begin{tabular}{|l|l|}
\hline Le patrimoine matériel & Le patrimoine immatériel \\
\hline $\begin{array}{l}\text { Couvents, temples, loges, portiques, autels } \\
\text { arbres sacrés, instruments et objets de culte }\end{array}$ & $\begin{array}{l}\text { Musiques, litanies, chants et danses sacrés, } \\
\text { rites et rituels, cérémonies, offrandes }\end{array}$ \\
\hline
\end{tabular}

31 Le culte vodoun a ceci de "commun" qu'il revêt une dimension historique et patrimoniale, et transcende bien souvent les différentes appartenances religieuses des individus: "Dans la famille, il y a des catholiques et des protestants, chacun fait ce qu'il veut, mais le culte, qui est l'héritage des ancêtres, reste toujours honoré ", raconte l'un des habitants. Les cultes et les pratiques ancestrales ont en effet survécu à l'adhésion au christianisme et 
à l'islam (Bassalé, 2014; Dorier-Apprill, 2006). Les hounwé, ou cérémonies vodoun, organisées périodiquement en l'honneur des divinités, mobilisent l'ensemble des habitants des places et au-delà (membres du lignage et habitants d'autres places vodoun de Porto-Novo). Le syncrétisme qui marque la ville semble conduire à une forme d'ouverture et de tolérance des communautés entre elles, même si quelques tensions entre communautés ont été révélées lors des entretiens.

\subsection{La dimension économique et sociale de ces espaces urbains}

L'appartenance et l'attachement aux places sont également liés à la vie sociale qui s'y développe. Au-delà de leur dimension cultuelle, les places vodoun revêtent en effet plusieurs fonctions socio-économiques. On y trouve divers types de commerces formels et informels: des vendeuses ambulantes et fixes proposent des en-cas et de la petite épicerie, un coiffeur y est domicilié. Ces places accueillent les événements privés et publics organisés par les habitants, par des acteurs locaux ou par la municipalité : cérémonies familiales, concerts, réunions publiques, ou encore rencontres politiques, etc. Ce sont également des lieux de médiation, où les familles se retrouvent pour échanger sur les projets liés au quartier, et régler les différends.

C'est aussi un lieu de détente et de loisirs pour les habitants et particulièrement pour les jeunes du quartier qui s'y retrouvent pour discuter, pratiquer une activité sportive ou jouer aux cartes. Ces fonctions sociales ont été renforcées à l'issue des travaux réalisés dans le cadre de l'opération "Éclosions urbaines", par de meilleures conditions d'éclairage et de sécurité, notamment la nuit, et par l'installation de mobilier urbain adapté aux usages des habitants.

\section{La mise en valeur des places, une démarche de co- construction de l'espace urbain}

Selon les témoignages recueillis, la prospérité des habitants est directement subordonnée au bien-être des divinités, et par conséquent à la qualité de la place. Aussi, l'adhésion au projet de réhabilitation des places s'explique tant par des problématiques tangibles de salubrité, de confort ou encore de sécurité, que par des préoccupations d'ordre spirituel: "Cette place contient nos vodoun, ils étaient là, mais ils n'étaient pas protégés, pas bien entretenus, il y avait des problèmes d'entretien par rapport aux bâtiments ", explique un habitant. Les travaux ont ainsi porté en priorité sur la réfection des murs et des façades des bâtiments et édifices cultuels, qui étaient dans un état de délabrement avancé - certains étant déjà écroulés -, mais pour les habitants, derrière les mauvaises conditions dans lesquelles étaient installées les divinités, c'est la perpétuation du culte lui-même qui était en jeu. La dimension spirituelle et symbolique de la place peut expliquer en partie la forte mobilisation des habitants autour du projet.

\subsection{La démarche participative, pour une œuvre commune}

Sur une place vodoun, la participation de la population n'est pas qu'un prétexte visant l'approbation du projet par les habitants : il s'agit d'un prérequis indispensable, une " nécessité conduite par la raison ", selon les termes de Gérard Bassalé, responsable du projet. 
En effet, en raison du caractère sacré et donc « sensible » de ces places, toute intervention sur ce type d'espace doit être validée au préalable par les chefs religieux et respecter un certain nombre de règles collectives à la fois formelles et informelles. Les aspects matériels mis en débat - plans d'implantation, couleurs des murs, emplacement des bancs ou encore motifs des fresques - renvoient ici à la dimension symbolique et spirituelle de la place. Ainsi, des réunions de concertation ont inclus, dans un ordre respectant les hiérarchies en présence, les chefs du culte vodoun, les représentants des familles et les responsables administratif ${ }^{19}$, puis les différentes composantes du quartier: les commerçants, les adeptes, les artisans et l'ensemble des habitants. Signalons qu'une vigilance particulière a été portée à la présence des femmes lors de ces rencontres, même si dans la réalité, les femmes semblent avoir été moins nombreuses que les hommes. Certaines personnes rencontrées n'ont pas été tenues au courant des dates, d'autres sont restées chez elles pour s'occuper de leur famille, s'appuyant sur la présence de leur mari aux réunions. Des visites collectives des temples et des couvents ont ensuite été organisées afin de recueillir la connaissance et l'expertise d'usage des habitants, et d'en tenir compte dans la définition des futurs aménagements. Le périmètre des personnes concertées ne s'est pas arrêté aux habitants de la place. Les membres des lignages familiaux vivant à l'extérieur, dans d'autres quartiers, voire d'autres villes, mais conservant un ancrage très fort sur les places ont en effet été mis au courant du projet. Cette concertation s'est également accompagnée d'un ensemble de rites consistant à consulter les divinités afin d'obtenir leur approbation. Après un recueil exhaustif des propositions des habitants, de nombreuses discussions ont été organisées. Animés de manière à favoriser la « co-construction " du projet, ces échanges ont permis de trouver des compromis entre les envies de la population et les contraintes financières et techniques.

\subsection{Les habitants, véritables acteurs de la réhabilitation}

Le projet a permis aux habitants de s'impliquer et d'être acteurs de la transformation de leur quartier, dans une démarche qui renvoie à la notion d'empowerment visant l'ensemble de la communauté. Cette démarche d'empowerment, qui désigne «le pouvoir d'agir », est censée donner aux habitants les moyens de s'impliquer dans les transformations urbaines, et plus largement de peser sur leurs destins (Bacqué et Biewener, 2013). À Porto-Novo, les habitants ont pris en charge différentes missions techniques et logistiques. La préparation et l'installation du chantier ont en effet nécessité une phase de désherbage, sarclage, élagage des arbres, nettoyage, balayage, acheminement et stockage des matériaux, qu'ils ont assurée eux-mêmes. L'exécution des travaux a mobilisé différents corps de métiers dans le domaine du bâtiment: maçons, peintres, soudeurs, coffreurs, menuisiers, ferrailleurs et manœuvres, en grande majorité recrutés parmi les habitants du quartier. D'autres tâches afférentes au chantier ont également été assurées par les membres des familles, à l'exemple du stockage et du gardiennage des matériaux. Par ailleurs, plusieurs habitants ont joué un rôle de médiation et d'encadrement pour assurer la bonne réussite des travaux. Dans le prolongement de l'opération de réhabilitation, une association chargée de l'entretien, du nettoyage et de la surveillance du quartier a été créée, composée d'habitants des places. 


\subsection{La création artistique, un catalyseur du commun?}

37 L'une des originalités de cette expérience de réhabilitation des places repose sur sa dimension artistique. Un réseau d'artistes contemporains a en effet été mobilisé pour contribuer à la transformation des espaces communs, à travers la création d'œuvres individuelles et collectives. Des œuvres permanentes ont été intégrées aux éléments architecturaux qui composent la place: murs des temples et des couvents, portes et enceintes des loges, escaliers, mobilier urbain, etc. En bas de l'escalier, face à la lagune, deux grandes œuvres des artistes Philippe Zountègni Houedanou et Simplice Ahouansou ont été installées. Par ailleurs, des peintures et sculptures sont présentées chaque année, pour une durée d'un an, dans la « rue des artistes », cheminement piétonnier aménagé à l'occasion des travaux. L'intervention des habitants a, cette fois encore, été essentielle. Ils ont tout d'abord accueilli les artistes pour présenter les places et les faire visiter. Ils ont ensuite exprimé leurs attentes vis-à-vis des œuvres à venir et formulé un certain nombre de points de vigilance. À partir de ces recommandations, les artistes ont travaillé sur des maquettes qui ont ensuite été soumises à la validation des habitants. Ces allers-retours ont permis de mettre en correspondance chaque œuvre avec les divinités locales.

Ces créations artistiques ne sont pas seulement "un supplément d'âme » du projet de réhabilitation de la place. Elles en font partie intégrante et jouent plusieurs fonctions. Elles contribuent à donner à voir un patrimoine immatériel la plupart du temps invisible. Les enjeux de conservation, de valorisation et de transmission d'un commun, qu'il convient de protéger et faire perdurer, sont au cœur du projet. Cette transmission vise en particulier les générations à venir, et les œuvres sont conçues pour permettre de les guider dans la connaissance du vodoun.

\subsection{Un commun, ouvert et partagé}

Après la concertation et les travaux, la troisième étape du projet consiste à mettre en place une stratégie globale de valorisation des places. Celle-ci s'appuie notamment sur le développement de circuits de "tourisme responsable ", c'est-à-dire organisés par et avec les habitants, et dont les bénéfices économiques seront réinjectés dans des projets utiles à la collectivité ${ }^{20}$. La mise en valeur du patrimoine par le tourisme s'est construite autour de la formation de guides locaux recrutés parmi les habitants du quartier, l'édition de documents explicatifs et l'instauration d'une billetterie, assurée par l'association en charge de la gestion des places, et dont les revenus doivent servir à assurer l'entretien du quartier. Il est difficile à ce stade de mesurer les retombées économiques de cette démarche qui a vu le jour en 2017.

Selon Monica Coralli et Didier Houénoudé (2013), "le patrimoine comme enjeu de développement local via le tourisme demeure une invention exogène dont les acteurs locaux s'accommodent, sauf exception, plus pour capter des financements que par conviction ». Si ce constat peut s'appliquer à Porto-Novo et si la dimension financière peut expliquer en partie la satisfaction exprimée par certains habitants, les entretiens montrent qu'il existe une réelle fierté face aux très nombreuses visites que reçoivent ces places depuis leur réhabilitation, et les personnes sont heureuses de pouvoir rendre visible, partager et transmettre leur culture. 
41 représentations souvent négatives liées aux pratiques vodoun, qui fascinent autant qu'elles terrifient, de par le côté violent de certains rituels: scarifications, sacrifices d'animaux, etc. La fierté exprimée semble être à la hauteur de la gêne vécue auparavant face à l'état de délabrement avancé de ces places.

\section{Conclusion}

Dans le prolongement de cette expérience, une cinquième place va être réhabilitée à Porto-Novo lors de l'édition 2018 du festival artistique Éclosions urbaines. Différents projets de valorisation sont d'ores et déjà envisagés afin de faire connaître au plus grand nombre l'existence des places vodoun de la ville. Celles-ci représentent en effet un patrimoine spécifique, par l'articulation marquée qui existe entre ses dimensions matérielles et immatérielles. Le caractère symbolique de ces places et leur rôle sur le plan familial, social, culturel et cultuel, contribuent à faire de ces espaces urbains de véritables communs. Au-delà, la démarche de réhabilitation articulant participation des habitants et implication des artistes plasticiens, illustre les approches mises en œuvre actuellement dans le cadre de la recommandation de l'Unesco sur le paysage urbain historique, visant à promouvoir un développement durable des villes.

\section{BIBLIOGRAPHIE}

Auclair E., Fairclough G. (eds), 2015, Theory and practice in heritage and sustainability : living between past and future, London, Routledge.

Auclair E., Hertozg A., Poulot M.-L. (dir), 2017, De la participation à la coconstruction des patrimoines, l'invention des communs?, Paris, Éditions Le Manuscrit, coll. » Devenirs urbains ».

Bacqué M.-H., Biewener C., 2013, L'empowerment, une pratique émancipatrice, Paris, La Découverte, coll. » Politique et sociétés ».

Bassalé G., 2013, « Enjeux des places vodoun dans l'évolution de la ville de Porto-Novo », Mengin C., Godonou A. (dir), 2013, Porto-Novo : patrimoine et développement, Paris, Publications de la Sorbonne.

Bassalé G., 2014, « Enjeux des places vodoun dans l'évolution des villes du Sud-Bénin. Cas de Porto-Novo ", mémoire de maîtrise d'histoire, sous la direction de Andande J. et Houénoudé D., université d'Abomey Calavi.

Bollier D., 2014, La renaissance des communs, Paris, Éd. Charles Léopold Mayer.

Boniburini I., Le Maire J., Moretto L., Smith H., 2013, « La ville comme bien commun :

planification urbaine et droit à la ville ", Bruxelles, Les Cahiers d'architecture La Cambre-Horta $n^{\circ} 9$.

Coralli M., Houénoudé D., 2013, « La patrimonialisation à l'occidentale et ses conséquences sur un territoire africain », Espaces et Sociétés, p. 152-153.

Développement durable et territoires, Vol. 10, n 1 | Avril 2019 
Coriat B. (dir), 2015, Le retour des communs. La crise de l'idéologie propriétaire, Paris, Les Liens qui libèrent.

Cornu M., Orsi F., Rochefeld J. (dir), Dictionnaire des biens communs, Paris, PUF.

Cousin S., 2013, «Extension du domaine de la restauration Porto-Novo capitale : entre vision patrimoniale, modernité vodoun et regard touristique », in Mengin C., Godonou A. (dir), 2013, Porto-Novo : patrimoine et développement, Paris, Publications de la Sorbonne.

Dardot P. et Laval C., 2014, Commun, essai sur la révolution au XXI siècle, Paris, La Découverte.

Dorier-Apprill E., 2006, « Les échelles du pluralisme religieux en Afrique subsaharienne », L'Information géographique, 2006/4 (vol. 70), p. 46-65. DOI 10.3917/lig.704.0046p

Dorier E., Tafuri C., Agossou N., 2013, « Porto-Novo dans l'aire métropolitaine littorale du SudBénin : quelles dynamiques citadines ? » in Mengin C., Godonou A. (dir), Porto-Novo : patrimoine et développement, Paris, Publications de la Sorbonne.

EPA, Institut français et Cité de l'architecture et du patrimoine, 2016, « Liaison urbaines, transformations d'espaces publics de villes africaines ».

Fabre D., 2013, Émotions patrimoniales, Paris, Éditions de la Maison des sciences de l'homme.

Fabre D. 2016, « L'ordinaire, le familier, l'intime, loin du mouvement », in Hottin C., Voisenat C. (dir), Le moment patrimonial. Mutations contemporaines des métiers du patrimoine, Paris, Éditions de la Maison des sciences de l'homme.

Gravari Barbas M. (dir), 2003, Habiter le patrimoine, Enjeux, approches, vécu, Rennes, PUR.

Harvey D., 2015, Villes rebelles, du droit à la ville à la révolution urbaine, Paris, Éditions Buchet/ Chastel.

Heinich N.,2009, La fabrique du patrimoine, de la cathédrale à la petite cuillère, Paris, Éditions de la Maison des sciences de l'homme.

Hertzog A., Poulot M.-L., Auclair E., 2017, « Poser les jalons d'un inventaire participatif : retour sur la complexité d'une démarche de coconstruction à Cergy-Pontoise », in Auclair E., Hertozg A., Poulot M.-L. (dir), De la participation à la coconstruction des patrimoines, l'invention des communs?, Paris, Éditions Le Manuscrit, coll. » Devenirs urbains ».

Juhé-Beaulaton D., 2009, « Un patrimoine urbain méconnu. Arbres mémoires, forêts sacrées et jardins des plantes de Porto-Novo (Bénin) », Autrepart 2009/3 ( ${ }^{\circ} 51$ ), p. 75-98. DOI 10.3917/ autr.051.0075

Le Crosnier H., 2015, En communs : une introduction aux communs de la connaissance, C. et F. Éditions.

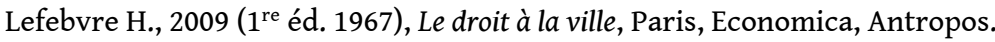

Leyronas S., Bambridge T. (dir), 2018, Lecture croisée de la gouvernance des communs, Revue internationale des études de développement 2018/1 n²33.

Magnaghi A., 2014, La biorégion urbaine, petit traité sur le territoire bien commun, Paris, Eterotopia, Rizhome.

Mengin C., 2013, « Introduction », in Mengin C., Godonou A. (dir), 2013, Porto-Novo : patrimoine et développement, Paris, Publications de la Sorbonne.

Mengin C., Godonou A. (dir), 2013, Porto-Novo : patrimoine et développement, Paris, Publications de la Sorbonne. 
N’Bessa B., 2013, « Le doublet Porto-Novo/Cotonou : un développement urbain conditionné par l'économie et la politique ", in Mengin C., Godonou A. (dir), Porto-Novo : patrimoine et développement , Paris, Publications de la Sorbonne.

Nussbaum M., 2011, Capabilités. Comment créer les conditions d'un monde plus juste ? Paris, Climats.

Oloudé B., 2003, Synthèse des débats, session organisée par l'Unesco dans la cadre du sommet Africités III, Yaoundé, 2 décembre 2003, p. 30, cité par Christine Mengin, in Mengin C., Godonou A. (dir), 2013, Porto-Novo : patrimoine et développement, Paris, Publications de la Sorbonne.

Ostrom E., 1990, Governing the Commons : the evolution of institutes for collective action, Cambridge, Cambridge University press.

Raimbault L., 2015, «Éclosions urbaines, le point de vue de l'urbaniste », in Éclosions urbaines. Révéler le réseau des places traditionnelles, pollen d'une urbanité africaine oubliée, édité par la Ville de Porto-Novo, la Communauté d'agglomération de Cergy-pontoise, Ouadada et l'École du patrimoine africain.

Rautenberg M., 2003, La rupture patrimoniale, Bernin, Éditions À la croisée.

Sgard A., 2010, «Le paysage dans l'action publique : du patrimoine au bien commun », Développement durable et territoires, vol. $1, \mathrm{n}^{\circ} 2$.

Sinou A., 2013, « Les singularités des formes patrimoniales à Porto-Novo », in Mengin C., Godonou A. (dir), Porto-Novo : patrimoine et développement, Paris, Publications de la Sorbonne Sinou A., Oloudé B.,1989, Porto-Novo, ville d'Afrique noire, Marseille, Éditions Parenthèses.

Tall E.K., 1995, « Dynamique des cultes voduns et du christianisme céleste au sud Bénin », Cahiers des sciences humaines, vol. $4, \mathrm{n}^{\circ} 31$.

Tall E.K., 2009, « Imaginaire national et mise en patrimoine dans l'Atlantique sud », Usotopie XVI (2).

Verger Fatumbi P., 1995, Dieux d'Afrique. Culte des orishas et vodouns à l'ancienne côte des esclaves en Afrique et à Bahia, la baie de tous les saints au Brésil, Paris, Revue noire.

Vernières M., 2013, Avant-propos, in Mengin C., Godonou A. (dir), Porto-Novo : patrimoine et développement, Paris, Publications de la Sorbonne.

Veschambre V., 2008, Traces et mémoires urbaines. Enjeux sociaux de la patrimonialisation et de la démolition, Rennes, PUR.

Vidégla M., 2013, « Les éléments sombres de l'histoire de Porto-Novo : esclavage et colonisation » in Mengin C., Godonou A. (dir), Porto-Novo : patrimoine et développement, Paris, Publications de la Sorbonne.

Watremez A., 2008, «Vivre le patrimoine urbain au quotidien : pour une approche de la patrimonialité », Culture \& Musées, $\mathrm{n}^{\circ} 11$.

\section{NOTES}

1. 260000 lors du recensement de 2013 ; l'agglomération compte environ un million d'habitants en 2017.

2. 760000 habitants selon le recensement de 2013 ; l'agglomération compte environ 2,5 millions d'habitants en 2017. 
3. Cf. document intitulé «Porto-Novo, éclosions urbaines. Révéler le réseau des places traditionnelles, pollen d'une urbanité africaine oubliée », publié en 2015 par la ville de PortoNovo, la CACP, le Centre culturel Ouadada et l'École du patrimoine africain.

4. Idem.

5. Le projet Porto-Novo - Ville Verte fait l'objet d'une convention entre la ville de PortoNovo, la CACP, la Métropole du Grand Lyon, l'AFD et le Fond français pour l'environnement mondial ; elle a été signée lors de la COP 21 à Paris en 2015 (pour un montant total de 9200000 euros).

6. Si les habitants peuvent être propriétaires de leur maison et de leur cour, il n'existe pas de titre de propriété, à proprement parler, pour l'espace central délimité par la place.

7. Un premier travail de terrain a eu lieu en janvier 2016, permettant de visiter et étudier l'ensemble des places traditionnelles de la ville, et de rencontrer les principaux responsables concernés par ce projet. Une seconde mission de terrain, réalisée en mai 2017, a permis la réalisation d'une cinquantaine d'entretiens avec des habitants et différents acteurs locaux (chefs religieux, responsables locaux, artistes, directeur du centre culturel, etc.). Les entretiens ont eu lieu en français, avec si nécessaire l'appui de traducteurs béninois. Deux autres missions menées au printemps 2018 ont permis de poursuivre les travaux et d'approfondir la recherche, dans le cadre d'une coopération signée entre l'UCP et l'université d'Abomey Calavi. Ce travail bénéficie de soutiens financiers de la part de la CACP et de l'ambassade de France au Bénin.

8. Un inventaire du patrimoine, réalisé en 2001-2002 par le l'EPA et l'École africaine des métiers de l'architecture et de l'urbanisme avec des financements de la Banque Mondiale, a identifié six grandes catégories de patrimoines : colonial, afro-brésilien, royal, lignager, religieux, paysager. Ce travail avait notamment pour objet de préparer une demande de classement au titre du patrimoine mondial de l'Unesco, lequel n'a pas été accepté jusqu'à présent.

9. La protection des berges lagunaires, dont la richesse des paysages, de la faune et de la flore est exceptionnelle, constitue un enjeu important pour la ville, comme en témoigne le projet PortoNovo - Ville verte.

10. La ville de Porto-Novo a initié le projet Éclosions urbaines. En outre, un musée du Vodoun à Porto-Novo est inscrit parmi les grands chantiers touristiques voulus par le gouvernement et mis en œuvre par l'Agence nationale de promotion des patrimoines et de développement du tourisme.

11. La position de l'église catholique a néanmoins évolué par la suite, vers une plus grande ouverture et tolérance vis-à-vis du vodoun (cf. Bassalé 2014). En revanche, l'église évangélique, très active dans la région, s'oppose fortement à ces pratiques (cf. Tall, 1995).

12. Dans les questionnaires utilisés pour les statistiques officielles relatives aux pratiques religieuses, les personnes ne peuvent déclarer qu'une seule religion.

13. Ceux qui sont "désignés", lors d'une cérémonie appelée le Fa, pour être "vodounsis ", sont les adeptes : ils entrent au couvent pour apprendre les rites, danses et chants mobilisés lors des cérémonies. Les femmes portent les épaules nues et des scarifications sont réalisées sur leurs épaules; les hommes restent torse nu.

14. Hysope, fromager, colatier, iroko, ficus...

15. Les legba sont de petits édifices sacrés qui contiennent un fétiche, lequel représente le support matériel d'une divinité.

16. Les zangbétos, dont la fonction est de maintenir le calme et la sécurité dans le quartier, relèvent de sociétés secrètes, « les sociétés de masques ».

17. Cf. ouvrage intitulé Liaison urbaines, édité en 2016, par l'EPA, l'Institut français et la Cité de l'architecture et du patrimoine. 
18. Au-delà de son expertise dans le domaine de la mise en valeur du patrimoine, la structure Ouadada propose différentes actions culturelles et touristiques (spectacles, ateliers, stages et cours, notamment avec les scolaires, expositions d'art plastique, circuits de tourisme culturel...). 19. Il s'agit ici des délégués de quartier, représentants de la municipalité sur les places.

20. Démarche qui se rapproche de celle des greeters qui se déploient dans de nombreux pays.

\section{RÉSUMÉS}

Les places traditionnelles de Porto-Novo sont des espaces urbains combinant des fonctions religieuses, culturelles, sociales et économiques. Ces places "communes", ni publiques ni privées, car appartenant symboliquement à une collectivité familiale, un lignage, illustrent l'articulation entre plusieurs dimensions: matériel/immatériel, mais aussi visible/invisible, public/privé, sacré/profane. L'analyse de ces places se base sur l'étude des perceptions et représentations formulées par les habitants ainsi que sur les dynamiques sociales générées par une opération de réhabilitation, de mise en valeur des patrimoines et de création artistique. La culture, la mémoire, les traditions, les pratiques culturelles sont autant de formes collectives d'appartenance et d'attachement pouvant être comprises comme les signes de la construction de communs.

The traditional places in Porto Novo are urban spaces which combine religious, cultural, social and economic functions. These places are neither entirely public nor private as they symbolically "belong" to the families, the communities who live there; they therefore can be considered as commons. They illustrate a complex combination between opposite dimensions such as material/ immaterial, sacred/secular, public/private, visible/invisible. This article examines the perceptions and representations of the inhabitants, the dynamics induced by the rehabilitation of the traditional places and the involvement of local artists. Culture, memories, traditions, and religious practices are elements which contribute to the place and sense of belonging, and participate in defining these places as commons.

\section{INDEX}

Keywords : commons, heritage, urban planning, art works, participation

Mots-clés : commun, patrimoine matériel et immatériel, patrimonialisation, aménagement urbain, création artistique, participation, co-construction

\section{AUTEURS}

\section{ELIZABETH AUCLAIR}

Elizabeth Auclair est docteur ès géographie, maître de conférences, HDR, en aménagement. Ses travaux portent principalement sur les dynamiques artistiques, culturelles et patrimoniales et sur les démarches participatives, en lien avec le développement soutenable des territoires. Elle est membre du laboratoire MRTE, université de Cergy-Pontoise département de géographie et 
histoire.

Elizabeth.auclair@u-cergy.fr

\section{ÉLISE GARCIA}

Élise Garcia est docteur en aménagement, maîtresse de conférences associée (PAST) et membre associée au laboratoire de géographie MRTE. À travers ses travaux, elle interroge les rôles et fonctions des actions internationales des collectivités territoriales et leurs effets en matière de développement des territoires. Université de Cergy-Pontoise département de géographie et histoire.

Elise.garcia@outlook.com 